connected to the main channel ${ }^{5}$. Prolonged inundation with floodwater can cause some species of tree - particularly oak - to form wood that has abnormal features. The annual growth rings of such trees in the 'bottomland' hardwood forest of the Mississippi floodplain therefore contain a natural record of past floods ${ }^{6}$. By splicing together sedimentary sequences from lakes in Louisiana and Mississippi with 'flood-ring' signatures from living and dead trees in southeastern Missouri, the authors assembled a flood chronology for the lower Mississippi that stretches back to the early sixteenth century.

Together, these natural archives have kept a remarkably faithful account of past floods. The tree rings mark the occurrence of the great floods of 1844 and 1927, as well as l'Année des Grandes Eaux (the Year of the Great Waters) in 1785, which destroyed French-Canadian settlements in Illinois and Missouri. It is more difficult to date individual floods in the lake records because of the lower chronological resolution of that archive, but layers of coarse sediment can be matched to the great floods of 1851, 1927 and 2011, as well as to the flood reported by Spanish conquistadors in 1543 . Overall, this new palaeoflood record suggests that, although flood hazards have waxed and waned through time, the Mississippi has risen higher and flooded more frequently in the past century than during any other period in the past 500 years.

The authors propose a provocative explanation for this recent hydrological intensification. Since the start of the twentieth century, records gathered using instruments show that the discharge of the Mississippi has slowly risen and fallen in concert with the surface temperature of the North Atlantic Ocean, which has alternated every two or three decades between warm and cold states ${ }^{7}$. Munoz and colleagues draw on proxy temperature estimates for the North Atlantic ${ }^{8}$ to demonstrate that this dependency has held steady since the 1500s. Because the spate of major floods in the past century cannot be explained by the observed temperature behaviour of the North Atlantic, the authors conclude that the trend towards larger and more frequent floods is mostly due to the transformation by humans of the Mississippi River and its basin.

Blaming floods on the infrastructure that was built to guard against them will be controversial. The Mississippi basin has undergone upward trends in precipitation and evapotranspiration - the sum of evaporation and plant transpiration from Earth's surface - in the past several decades ${ }^{9}$, so climatic factors other than the influence of the North Atlantic might also have affected the rhythm of the river. And, like the rest of North America, the Mississippi basin has warmed substantially since the end of the Little Ice Age ${ }^{10}$ (a period of cooling that began in the sixteenth century and ended in the mid-nineteenth century ${ }^{11}$ ), so
I think it is possible that the long-term trends in hydrology could be the result of climate change, rather than river engineering. Testing these competing explanations will require more palaeoflood work to be performed along the upper Mississippi and its main tributaries, where modifications to the river are less intensive and the climate still dominates the river's hydrology ${ }^{12}$.

In the meantime, Munoz and co-workers' study makes it clear that a century or so of hydrological readings is not sufficient to take the measure of a river such as the Mississippi. Their palaeoflood record is compelling because it offers an opportunity to step back and consider the ebb and flow of the river on a timescale that befits its majesty. And if the authors are correct, and collective efforts to subdue the Mississippi have inadvertently pushed it to rise higher than ever, then the time might have come to consider loosening its restraints. .

Scott St. George is in the Department of Geography, Environment and Society,
University of Minnesota, Minneapolis,

Minnesota 55455, USA.

e-mail:stgeorge@umn.edu

1. Munoz, S. E. et al. Nature 556, 95-98 (2018).

2. Pinter, N., Jemberie, A. A., Remo, J. W. F. Heine, R. A. \& Ickes, B. S. Geophys. Res. Lett. 35, L23404 (2008).

3. Watson, C. C., Biedenharn, D. S. \& Thorne, C. R. J. Hydraul. Eng. 139, 1071-1078 (2013).

4. Barry, J. M. Rising Tide: The Great Mississippi Flood of 1927 and How it Changed America (Simon \& Schuster, 1998).

5. Toonen, W. H. J., Winkels, T. G., Cohen, K. M. Prins, M. A. \& Middelkoop, H. Catena 130, 69-81 (2015).

6. Therrell, M. D. \& Bialecki, M. B. J. Hydrol. 529, 490-498 (2015).

7. Enfield, D. B., Mestas-Nuñez, A. M. \& Trimble, P. J. Geophys. Res. Lett. 28, 2077-2080 (2001).

8. Gray, S. T., Graumlich, L. J., Betancourt, J. L. \& Pederson, G. T. Geophys. Res. Lett. 31, L12205 (2004).

9. Milly, P. C. D. \& Dunne, K. A. Geophys. Res. Lett. 28, 1219-1222 (2001).

10.Trouet, V. et al. Environ. Res. Lett. 8, 024008 (2013).

11.Matthews, J. A. \& Briffa, K. R. Geogr. Ann. A 87, $17-36$ (2006).

12.Wise, E. K., Woodhouse, C. A., McCabe, G. J., Pederson, G. T. \& St-Jacques, J.-M. J. Hydrometeorol. 19, 161-182 (2018).

\title{
Forests in flux as climate varies
}

\section{How do changes in climate affect forest ecosystems? A study of temperate forests in the United States has assessed alterations in biomass and tree-species composition across a 20-year period of climate variability. SEE LETTER P.99}

\section{SEBASTIAAN LUYSSAERT} \& J. HANS C. CORNELISSEN

$\square$ ocumenting and understanding changes induced by climate in the composition and function of vegetation is essential for planning adaptation strategies, because chances to intervene do not arise often for forests that contain trees with long lifespans. Moreover, most of the effects exerted by climate change on the composition and biomass of vegetation probably occur incrementally rather than abruptly ${ }^{1}$, which makes their detection a challenge. On page 99, Zhang et al. ${ }^{2}$ report an analysis of changes in forest biomass in the eastern United States over two decades, during a time when some regions became drier and others became wetter.

Ecological studies have long focused on analyses in which the main groupings for organisms being studied are determined by evolutionary relationships such as belonging to the same genus. However, such kinship can hide functional differences. For example, even among closely related species of oak tree (Quercus spp.), some will thrive under moist conditions, whereas others are more suited to dry climates. In the past decade, the use of functional rather than kinship-driven approaches to grouping has provided many important insights ${ }^{3}$, and Zhang and colleagues' work can be added to the list of studies that have successfully done this.

Zhang et al. sought to investigate whether shifts in climate affect forest characteristics such as the prevalence of drought-tolerant species and the total biomass of the tree population. The authors compared temperateforest inventory data ${ }^{4}$ gathered during the 1980 s and the 2000s. This inventory includes surveys of around 100,000 plots, in which data such as species name and a standardized measurement of tree diameter were recorded for roughly 3 million trees. Diameter measurements allow the amount of biomass that is present above the surface of the ground to be estimated for a particular tree on the basis of previous studies of growth patterns for 


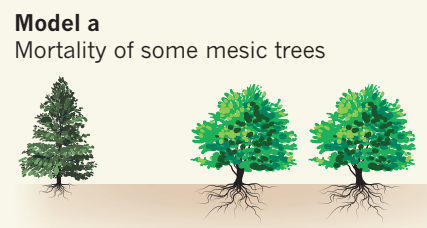

Model b

Drought-tolerant-tree growth is less

Mesic Drought-tolerant

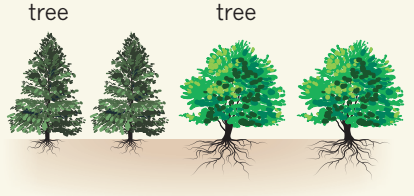

Forests reassessed 20 years later, after a period of drought
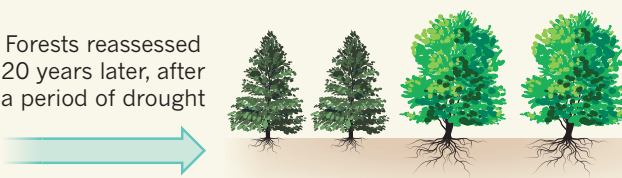

1980s
Model c

Sapling established

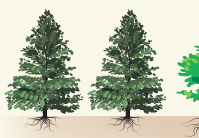

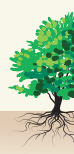

2000s

Figure 1 | Changes in the drought tolerance of tree populations. Zhang et al. ${ }^{2}$ used an inventory ${ }^{4}$ of trees growing in the eastern United States in the 1980s and the 2000s to estimate how an intervening period of drought had affected the contribution of drought-tolerant species to total biomass, in forests that had reached a given age. This analysis revealed an increase in the contribution of drought-tolerant trees compared with that of moisture-needing (mesic) trees to the population-level biomass. Three models could account for such an increase. Model a is consistent with the results for forests composed of trees more than 80 years old. Model b can account for the patterns observed by the authors in most other forests. The study's time span is probably too short to fully capture the sapling emergence of model c. The drought tolerance of trees might partly depend on their entering into symbiotic interactions with root-colonizing fungi that extend the effective root length ${ }^{9}$. Such interactions could reduce tree biomass. If so, this might need to be considered in studies of this type.

a given species. Such analyses enabled the authors to assess the aboveground biomass per hectare for tree populations, and also to investigate whether changes occurred in the relative contribution of particular types of species, such as drought-tolerant trees, to the total aboveground biomass.

The authors analysed the data grouped into grids of cells that each covered an area of one degree of latitude by one degree of longitude (about 110 kilometres by 85 kilometres). The plots sampled in the 1980s and the 2000s were not identical; however, the authors were able to check their findings using a subset of plots that had been resampled and found that their conclusions remained the same. The authors determined the tree-age composition of the forests and assigned them into 20 -year-interval age brackets. This enabled comparisons to be made between similar types of forest, for example, comparing 20-40-year-old forests in the 1980s and in the 2000s, therefore avoiding possible confounding factors such as changes in drought sensitivity that are linked to the increase in tree height as trees age $e^{5}$.

The authors assigned a numerical score to each recorded species of tree that represented its drought-tolerant characteristics. This score was generated on the basis of information about water availability, derived from measurements of the annual precipitation at grid cells where the species was found, and the minimum water requirements of that species. For each plot, the authors calculated the average drought tolerance of its tree population by using the species' drought scores, also taking into account the relative abundance of each species. The authors then compared how drought tolerance at the tree-population level for a particular age class in a given grid cell changed in the roughly 20 years between inventories, and assessed whether these changes mirrored any changes in soil moisture (estimated by the Palmer drought severity index) at each location.

Despite the occurrence of unavoidable problems such as logging during the datacollection period, Zhang and colleagues build a strong case that, between the 1980s and the 2000 s, the effects of ongoing climate variation in this zone of modest regional climate change have resulted in detectable changes in forest composition and biomass. In areas in which soil moisture increased over time, the authors observed a population-level decrease in drought tolerance and a population-level increase in aboveground biomass per hectare.

The most striking finding made by Zhang and colleagues was that, in areas in which soil moisture had decreased, a decrease in the average aboveground biomass gained per tree was accompanied by an increase in population-level drought tolerance. This increased share of drought-tolerant species occurred because the decrease in the growth rate of moisture-needing (mesic) species during a drought is greater than the decrease in growth rate of drought-tolerant species. Consequently, the forests that reached the particular age range in the 2000s have a lower biomass and a larger proportion of drought-tolerant species than the forests that reached this age range in the 1980s.

In forests containing trees more than 80 years old that experienced drought, the population-level changes in drought tolerance observed by the authors were often driven by an increase in the mortality of mesic species (model a in Fig. 1). The role of preferential tree mortality in driving forest changes that are linked to climate variability has already been reported in a study in Europe ${ }^{6}$. Yet, for most age ranges, the authors found that the tree population became more tolerant to drought because the growth of the drought-tolerant species was less affected than was that of the mesic species. This means that the droughttolerant trees increased their proportional contribution to the aboveground biomass per hectare in the study's two-decade period (model b in Fig. 1).

Although the establishment of saplings probably contributes to the response of forests to climate variability (model c in Fig. 1), the short time span of the study, the fact that forest inventories commonly measure only individual trees above a threshold size that it can take a decade or more for a sapling to reach, and the relatively small contribution of saplings to the total aboveground biomass for a given hectare of established forest, make it unlikely that Zhang and colleagues' approach would capture fully the changes in species composition that are due to sapling emergence.

The authors suggest that their results and observations could have relevance for how climate is affecting other temperate forests or forests in other climate zones. Yet perhaps the priority should be to unravel the mechanisms that underlie the connection between drought tolerance and biomass production at the species level. Zhang and colleagues suggest that the low availability of water should favour tree species that allocate a greater proportion of their biomass to fine roots, thereby promoting drought tolerance at the expense of aboveground biomass production. However, this might be only part of the climate-response phenomenon.

We speculate that, compared with mesic species, drought-tolerant species might have greater investments in symbiotic relationships with soil-dwelling mycorrhizal fungi that can colonize tree roots. Such interactions can extend the effective total root length, thereby extending access to water and nutrients 
in the soil ${ }^{7}$. However, such connections would probably come at a substantial cost in terms of tree-biomass reduction because of the need to divert sugars to fungal partners. The type and abundance of mycorrhizal symbioses vary with soil type and climate ${ }^{8,9}$, so if fungal symbiosis is a major consideration in these scenarios, such factors would need to be considered in future implementations of the approach used by Zhang and colleagues.

There is a pressing need to understand the relationship between water availability and the drought tolerance and biomass of forests. It is necessary, therefore, to ask whether the types of change that the authors observed would be able to keep pace with climate changes that occur on longer timescales. For example, will the drought-tolerance capacity of today's saplings suffice for the conditions that these trees might encounter when they reach maturity? It's high time to knock on wood that it will, as well as to continue to investigate the mechanisms that affect forest ecosystems in a changing climate.

\section{Sebastiaan Luyssaert and J. Hans C.} Cornelissen are in the Department of Ecological Science, Faculty of Science, VU Amsterdam, 1081 HV Amsterdam, the Netherlands.

e-mails: s.luyssaert@vu.nl;

j.h.c.cornelissen@vu.nl
1. Parmesan, C. \& Yohe, G. Nature 421, 37-42 (2003)

2. Zhang, T., Niinemets, Ü., Sheffield, J. \& Lichstein, J. W. Nature 556, 99-102 (2018)

3. Kunstler, G. et al. Nature 529, 204-207 (2016)

4. Bechtold, W. A. \& Patterson, P. L. The Enhanced Forest Inventory and Analysis Program - National Sampling Design and Estimation Procedures. Gen. Tech. Rep. SRS-80 (US Department of Agriculture Forest Service, 2005).

5. McDowell, N. G. \& Allen, C. D. Nature Clim. Change 5, 669-672 (2015).

6. Ruiz-Benito, P. et al. Glob. Change Biol. 23, 4162-4176 (2017)

7. Smith, S. E. \& Read, D. J. Mycorrhizal Symbiosis (Elsevier, 1997)

8. Phillips, R. P., Brzostek, E. \& Midgley, M. G. New Phytol. 199, 41-51 (2013).

9. Soudzilovskaia, N. A. et al. Glob. Ecol. Biogeogr. 24, 371-382 (2015).

This article was published online on 21 March 2018.

\section{Novel electronic states seen in graphene}

A simple system made from two sheets of graphene has been converted from an insulator to a superconductor. The finding holds promise for opening up studies of an unconventional form of superconductivity. SEE ARTICLE P.43 \& LETTER P.80

\section{EUGENE J. MELE}

$\mathrm{I}$ $\mathrm{n}$ two papers in Nature, Cao et al. ${ }^{1,2}$ report the discovery of new electronic ground states in twisted bilayer graphene - a pair of single-atom-thick sheets of carbon atoms, stacked with their honeycomb lattices rotated out of alignment. The authors interpret one of these states ${ }^{2}$ (page 80) as a correlated Mott insulator, a non-conducting state produced by strong repulsive interactions between electrons. The other ${ }^{1}$ (page 43) is a superconductor, a state of zero electrical resistance produced by effective attractive interactions between electrons. The insulator turns into the superconductor when a small number of charge carriers are added to the graphene. This connection between the states is unlikely to be a coincidence - as Sherlock Holmes might have commented, "the universe is rarely so lazy".

Cao et al. show that the stacking of graphene sheets allows access to a new family of materials with electronic behaviours that are exquisitely sensitive to the atomic alignment between the layers, which affects interlayer electron motion. This finding might surprise physicists, because electronic behaviour is usually dominated by whichever of the associated processes has the largest energy scale. But, in this case, there's a conundrum: the energy associated with electron motion between atoms within a layer is of the order of electronvolts, whereas the energy for electron motion between layers ${ }^{3}$ is, at most, hundreds of millielectronvolts.

The resolution to this conundrum is a matter of symmetry. Well-prepared, single layers of graphene are highly ordered systems whose electronic properties are determined by a subtle symmetry, which is encoded in a solidstate version of the Dirac equation describing low-energy excitations. These excitations are sensitive to interlayer couplings that alter the symmetries of the stack.

Interactions between electrons in these excitations can produce forms of matter generically described as being strongly correlated. A well-reasoned strategy for discovering such forms of matter has been to restrict intralayer electron motion by applying a strong magnetic field ${ }^{4}$. This generates narrow electron energy bands (Landau levels) in which electron-electron repulsion can control the physics of the graphene bilayer.

Cao et al. have taken a simpler tack to discover strongly correlated states. They used the rotational misalignment of graphene sheets to tune twisted bilayer graphene into a regime in which interactions between electrons can dominate the electronic states of the system. Such rotational misalignment forces the electronic band structures in the two sheets out of alignment and enlarges the bilayer's unit cell (the smallest repeating unit of the crystal lattice) (Fig. 1a). For large rotations, the first

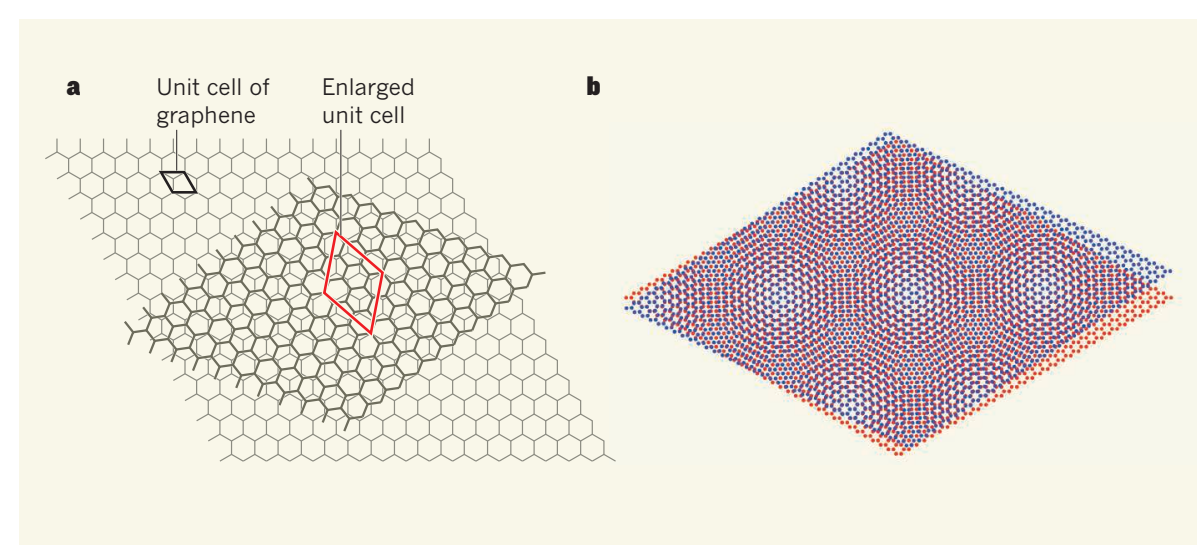

Figure 1 | The effects of rotation in twisted bilayer graphene. a, When a graphene bilayer is twisted so that the top sheet is rotated out of alignment with the lower sheet, the unit cell (the smallest repeating unit of the material's 2D lattice) becomes enlarged. For large rotations, the electronic band structures of the two graphene sheets are also rotated out of alignment (not shown). b. For small rotation angles, a 'moiré' pattern is produced in which the local stacking arrangement varies periodically. Cao et al. ${ }^{1,2}$ have observed that, for rotation angles of less than $1.05^{\circ}$, regions in which the atoms are directly above each other (the lighter regions in the pattern) form narrow electron energy bands, in which electron 'correlation' effects are enhanced. This results in the generation of a non-conducting state (a Mott insulator), which can be converted into a superconducting state ${ }^{1}$ if charge carriers are added to the graphene system. 